\title{
Bone Health in Premenopausal Chinese Patients after Adjuvant Chemotherapy for Early Breast Cancer
}

\author{
Claudia H.W. Yip ${ }^{a}$ Giok S. Liem ${ }^{a}$ Frankie K.F. Mo ${ }^{a, b} \quad$ Elizabeth Pang ${ }^{a, b}$ \\ Yuan-yuan Lei ${ }^{a}$ Leung Li ${ }^{a} \quad$ Christopher C.H. Yip ${ }^{a}$ Jane Koha,b Rita Y.W. Nga \\ Joyce J.S. Suen ${ }^{a}$ Winnie Yeo ${ }^{a, b}$ \\ ${ }^{a}$ Department of Clinical Oncology, Faculty of Medicine, Prince of Wales Hospital, The Chinese University of Hong \\ Kong, Shatin, Hong Kong SAR; ${ }^{b}$ State Key Laboratory of Translational Oncology, Faculty of Medicine, The Chinese \\ University of Hong Kong, Shatin, Hong Kong SAR
}

\section{Keywords \\ Osteoporosis · Osteopenia · Cytotoxic treatment}

\begin{abstract}
Background: In this cohort study, the objectives were to determine bone mineral density (BMD) and potential associated factors for bone health among young premenopausal patients after adjuvant chemotherapy. Methods: Eligibility criteria included premenopausal Chinese aged $<45$ years who had received adjuvant chemotherapy. At study entry, background demographics and menstrual history were collected; BMD was measured. Factors associated with reduced BMD and fracture risk were analyzed. Results: A total of 271 patients entered the study. The median time from breast cancer diagnosis to study entry was 5.0 years. The median ages at breast cancer diagnosis and at study entry were 41 and 47 years, respectively. The median BMDs for femoral neck (FN) and lumbar spine (LS) were 0.72 and $0.91 \mathrm{~g} / \mathrm{cm}^{2}$, respectively; $40.2 \%$ had abnormal Z-scores (defined as $\leq-1$ ) and $50.2 \%$ had osteopenia/osteoporosis of either FN or LS. On multivariate analyses, factors that were identified to have a positive association with bone health (higher BMD) included higher family income (OR [95\% Cl] for LS = 1.573 [1.0912.268]), taller stature (OR for LS = 2.975 [1.723-5.137]), and higher BMI (OR for FN = 2.156 [1.599-2.907]), while nega-
\end{abstract}

tively associated factors included longer interval since last adjuvant treatment (OR for LS: 0.435 [0.250-0.757]), peri-/ postmenopausal status at study entry (OR for LS $=0.443$ [0.255-0.768]; OR for $\mathrm{FN}=0.353$ [0.205-0.609]), and having received adjuvant tamoxifen (OR for $\mathrm{FN}=0.452[0.243-$ 0.841]). Conclusion: About 5 years after breast cancer diagnosis and adjuvant chemotherapy, $>50 \%$ of premenopausal patients who had received adjuvant chemotherapy were detected to have osteopenia/osteoporosis and $40 \%$ had abnormal Z-scores for FN/LS.

() 2020 S. Karger AG, Basel

\section{Background}

Breast cancer is the most common female malignancy in China, with an age-standardized rate of 27.96 per 100,000 population [1]; it is also the commonest female cancer in Hong Kong, with a relatively higher corresponding figure of 64.6 per 100,000 population. Over $80 \%$ of newly diagnosed breast cancer patients have earlystage disease [2]. For women with early-stage breast cancer who have undergone curative surgery, adjuvant therapies have shown to improve disease-free and overall survival; for the latter, it has been noted that young patients have an increased probability of receiving adjuvant cyto- 
toxic chemotherapy [3]. Adjuvant chemotherapy is well known to be associated with immediate as well as longterm toxicities, which may affect the quality of life and well-being of cancer survivors [4-7]. One of the chemotherapy-related long-term toxicities among premenopausal patients is hormonal changes with ovarian failure, which may lead to chemotherapy-related amenorrhoea (CRA) and early menopause. In our earlier report on young premenopausal Chinese patients after adjuvant chemotherapy, over $90 \%$ were reported to have developed CRA, while one-third subsequently developed menopause before the age of 45 [8].

Oestrogen deficiency as a result of ovarian dysfunction is a key factor that can lead to accelerated bone loss and subsequent increased incidences of fractures [9]. Earlier studies had reported that premenopausal breast cancer patients who received CMF chemotherapy (consisting of cyclophosphamide, methotrexate, and fluorouracil) and subsequently entered menopause had lower bone mineral density (BMD) when compared to those who did not develop menopause [10-15]. Other studies on women who had received relatively modern chemotherapeutic regimens including anthracyclines and taxanes had reported mean bone loss of 5\% in the lumbar spine (LS) over a 12-month period [16-19]. These findings have been based on Caucasian patient populations and are limited by small patient numbers, with most studies involving less than 50 patients; in addition, all apart from one study [20] had short follow-up of 1-2 years post-chemotherapy. Further, while data on BMD with or without Tscores have been commonly reported, data on Z-score for premenopausal breast cancer patients after adjuvant chemotherapy has been limited. Z-score has been regarded as a more accurate parameter for comparison as it represents the standard deviation (SD) in BMD relative to the expected BMD range for women of similar age, and is recommended by the International Society for Clinical Densitometry as the preferred assessment parameter for BMD in premenopausal women $[21,22]$.

In this cohort study on Chinese women with early breast cancer in Hong Kong, the objectives were to (1) determine the BMD, Z-scores, and T-scores based on dual energy X-ray absorptiometry (DXA) scanning among young premenopausal breast cancer patients after adjuvant chemotherapy, and (2) identify potential factors associated with these parameters.

\section{Patients and Methods}

Between September 2008 and February 2011, eligible breast cancer patients who were attending the breast cancer follow-up clinic at the Prince of Wales Hospital were approached for study enrolment. Eligibility criteria included female of Chinese ethnicity, a history of stage I-III breast cancer within 3-10 years, and pre- menopausal with age younger than 45 years when receiving adjuvant chemotherapy at the time of breast cancer diagnosis. Patients were excluded if they had evidence of disease recurrence. Patients who received ovarian ablation as part of the endocrine therapy or had hysterectomy prior to breast cancer diagnosis were also excluded. Eligible patients identified during their follow-up visits consented to the study. The study was approved by the Joint CUHK-NTEC Clinical Research Ethics Committee of the Chinese University of Hong Kong and Hong Kong Hospital Authority.

\section{Data Collection}

Clinical details at the time of breast cancer diagnosis were retrieved from individual patient's medical records. These included patient's age at breast cancer diagnosis, tumour characteristics, and information on treatment she received for her breast cancer (type of breast surgery, details of adjuvant radiotherapy, chemotherapy, tamoxifen and trastuzumab, and history of corticosteroid premedication during chemotherapy). Individual patient's body height and weight, measured by clinic staff at the time of diagnosis and at study entry, were also retrieved from the medical records. At study entry, patients' demographics (education level, employment history, level of family income, smoking and alcohol history, family history of breast cancer, and number of live births before breast cancer diagnosis) as well as medical history were collected. Each patient was asked to complete a study questionnaire in which they recalled their menstruation history with the assistance of a research assistant within the same hospital visit; details of the questions asked have been described in a previous report [8].

BMD (grams per square centimeter) measurements of the LS and femoral neck (FN) were obtained by DXA using Hologic Delphi W (S/N 70354) system. Z- and T-scores were calculated with the Hologic manufacturer's reference ranges for LS and FN.

\section{Definitions}

CRA was defined as amenorrhoea for $\geq 3$ months during and within 12 months after the completion of adjuvant chemotherapy [8]. Menopause was defined in line with World Health Organization (WHO) criteria as 12 months of amenorrhoea with last menstrual period $\geq 12$ months after chemotherapy and before study entry [23].

Body mass index (BMI) was categorized according to the WHO criteria for Asians. BMI categories included underweight, normal weight, overweight, and obesity, which were respectively defined as $<18.5,18.5-<23,23-<25$, and $\geq 25.0$ [24].

$\mathrm{Z}$-score is the number of SDs away from the mean bone density of a person of the same age, race, and gender [21]. For the purpose of this study, Z-scores of less than or equal to - 1 were defined as abnormal. T-score is defined as the number of SDs away from the mean bone density of a young adult with peak bone density. Osteoporosis was defined by the WHO [25] as a BMD below a Tscore of 2.5 SDs below the mean value of peak bone mass expected for a young healthy female adult either in the spine and/or the hip. Osteopenia was defined as a T-score between -1 and -2.5 SD.

Fracture risk of individual patients was being assessed by adopting Fracture Risk Assessment Tool $\left(\right.$ FRAX $^{\circledR}$ ) for Chinese [26]. This tool incorporates multiple factors including BMD of FN, based on which the 10-year probability of major osteoporotic hip fracture was calculated.

\section{Statistical Analysis}

Statistical analysis was performed by SAS version 9.3. Clinical characteristics data were summarized as patient number $(n)$ and percentage (\%) for categorical variables, and mean and SD for continuous variables. 
Table 1. Patients' background demographic and clinical characteristics at the time of breast cancer diagnosis $(n=271)$

\begin{tabular}{|c|c|c|}
\hline & Patients & $\%$ \\
\hline \multicolumn{3}{|l|}{ Age at diagnosis } \\
\hline$\leq 35$ years & 39 & 14.4 \\
\hline $36-40$ years & 81 & 29.9 \\
\hline $41-45$ years & 151 & 55.7 \\
\hline \multicolumn{3}{|l|}{ Age at time of study entry } \\
\hline$\leq 40$ years & 32 & 11.8 \\
\hline $41-45$ years & 74 & 27.3 \\
\hline $46-50$ years & 142 & 52.4 \\
\hline$>50$ years & 23 & 8.5 \\
\hline \multicolumn{3}{|l|}{ Education } \\
\hline Primary & 44 & 16.3 \\
\hline Secondary & 181 & 67.0 \\
\hline Tertiary & 27 & 10.0 \\
\hline Higher qualification & 18 & 6.7 \\
\hline \multicolumn{3}{|l|}{ Marital status } \\
\hline Single & 42 & 15.5 \\
\hline Married/partner & 209 & 77.1 \\
\hline Divorced/separated & 15 & 5.5 \\
\hline Widowed & 5 & 1.9 \\
\hline \multicolumn{3}{|l|}{ Employment } \\
\hline Full-time employment & 124 & 45.8 \\
\hline Part-time employment & 44 & 16.2 \\
\hline Unemployed/retired & 103 & 38.0 \\
\hline \multicolumn{3}{|l|}{ Family income* } \\
\hline$<$ HKD 5,000 & 26 & 9.6 \\
\hline HKD 5,000-25,000 & 143 & 52.8 \\
\hline HKD $25,000-50,000$ & 83 & 30.6 \\
\hline HKD 50,000 & 19 & 7.0 \\
\hline Weight at diagnosis $\geq$ median & 136 & 50.2 \\
\hline Height at diagnosis $\geq$ median & 138 & 50.9 \\
\hline Weight at study entry $\geq$ median & 140 & 51.7 \\
\hline Height at study entry $\geq$ median & 139 & 51.3 \\
\hline \multicolumn{3}{|l|}{ BMI at study entry (according to HK BMI) } \\
\hline Underweight $(<18.5)$ & 11 & 4.1 \\
\hline Normal (18.5-22.9) & 120 & 44.2 \\
\hline Overweight (23.0-24.9) & 59 & 21.8 \\
\hline Obese $(>25)$ & 81 & 29.9 \\
\hline \multicolumn{3}{|l|}{ Stage of breast cancer } \\
\hline Stage I & 86 & 31.8 \\
\hline Stage II & 160 & 59.0 \\
\hline Stage III & 25 & 9.2 \\
\hline \multicolumn{3}{|l|}{ Breast surgery } \\
\hline Lumpectomy & 94 & 34.7 \\
\hline Mastectomy & 177 & 65.3 \\
\hline Axillary lymph node dissection & 267 & 98.5 \\
\hline Received adjuvant radiotherapy & 180 & 66.4 \\
\hline \multicolumn{3}{|l|}{ Adjuvant chemotherapy regimen } \\
\hline Anthracycline-containing & 177 & 65.3 \\
\hline Anthracycline- and taxane-containing & 72 & 26.6 \\
\hline Others (taxane-containing, non-anthracycline + non-taxane containing) & 22 & 8.1 \\
\hline \multicolumn{3}{|l|}{ Adjuvant chemotherapy regimen } \\
\hline Taxane-containing & 77 & 28.4 \\
\hline Non-taxane-containing & 194 & 71.6 \\
\hline Duration of adjuvant chemotherapy $>64$ days & 184 & 67.9 \\
\hline \multicolumn{3}{|c|}{ Time since last adjuvant treatment (only include chemotherapy/radiotherapy or trastuzumab) } \\
\hline 3 to $<5$ years & 153 & 56.5 \\
\hline 5 to $<10$ years & 118 & 43.5 \\
\hline Ever received adjuvant tamoxifen & 205 & 75.7 \\
\hline On adjuvant tamoxifen at study entry & 110 & 40.6 \\
\hline
\end{tabular}


Table 1 (continued)

\begin{tabular}{lrr}
\hline & Patients & \multicolumn{1}{c}{$\%$} \\
\hline Received adjuvant trastuzumab & 8 & 3.0 \\
Use of traditional Chinese medicine since diagnosis & 80 & 29.5 \\
Having experienced chemotherapy-related amenorrhoea & 247 & 91.1 \\
Menopausal status at study entry & 109 & 40.2 \\
$\quad$ Premenopausal & 162 & 59.8 \\
$\quad$ Peri-/postmenopausal & 91 & 33.6 \\
Menopausal before age $<45$ for "postmenopausal” patients & & \\
\hline
\end{tabular}

* HKD, Hong Kong dollars; HKD 1 is equivalent to USD 0.128.

Baseline continuous variables were compared by Student's $t$ test or Mann-Whitney U test as appropriate, and categorical variables were compared by chi-square test.

Univariate logistic regression was performed to identify any potential factors associated with $\mathrm{BMD}, \mathrm{Z}$-score, and T-score postchemotherapy. The odds ratio (OR) and corresponding 95\% confidence interval (CI) were provided. Stepwise multivariate logistic regression analysis that included significant factors was conducted.

All statistical tests were two-sided, and $p$ values less than 0.05 were regarded as significant.

\section{Results}

In total, 300 breast cancer patients were approached for study entry, 14 of them declined participation. As a result, 286 patients consented to participate in this study. Two patients failed to meet inclusion criteria as they received neoadjuvant therapy for their stage IIIb breast cancers. Four patients withdrew with the reason that they did not have time to perform the study tests after consent. As a result, 280 eligible patients entered the study, amongst whom 271 underwent DXA assessment. Table 1 shows the patients' background demographics, tumour characteristics, and anticancer treatments received at breast cancer diagnosis.

The median age at breast cancer diagnosis was 41 years (range: 24-45). Adjuvant chemotherapy regimens included anthracycline-containing (65.3\%), anthracyclinetaxane-containing (26.6\%), taxane-containing (1.8\%), and non-anthracycline/non-taxane-containing (6.3\%) regimens. Two hundred and five patients $(75.7 \%)$ also received adjuvant tamoxifen; 180 (66.4\%) received adjuvant radiotherapy.

The median time from breast cancer diagnosis to study entry was 5.04 years (range: 2.96-9.94). The median age at study entry was 47 years (range: $28-54$ ). At the time of the study, $110(40.5 \%)$ patients were still on adjuvant tamoxifen therapy.
Two hundred and forty-seven of the 271 patients (91.1\%) had experienced CRA; the median period of CRA was 9.0 months. Of the 247 who had CRA, 82 did not regain their menstruation and went into menopause; for the remaining two-thirds who resumed their menstruation, 45 developed menopause, 95 remained to be premenopausal, and 25 were considered to be peri-menopausal at study entry. Of the 24 patients who did not experience CRA, 3 subsequently became peri-menopausal and 7 developed menopause. Thus, at study entry, 28 were peri-menopausal and 134 patients were postmenopausal; of the latter, $68 \%$ were younger than 45 at the time of menopause.

Body Weight, Body Height, and BMI at Breast Cancer Diagnosis and at Study Entry

At breast cancer diagnosis, the median weight and height of patients were $54.6 \mathrm{~kg}$ (range: 39.0-89.0) and 159 $\mathrm{cm}$ (range: 141-175), respectively; at study entry, the corresponding figures were $56.2 \mathrm{~kg}$ (range: $39.5-92.6$ ) and $157 \mathrm{~cm}$ (range: 143-172), respectively. At study entry, $4.1 \%$ were underweight, $44.2 \%$ were normal, $21.8 \%$ were overweight, and the remaining $29.9 \%$ were obese.

\section{Bone Health Assessment with DXA}

The median BMD of FN was $0.72 \mathrm{~g} / \mathrm{cm}^{2}$ (interquartile range [IQR]: $0.66-0.70$ ), that of LS was $0.91 \mathrm{~g} / \mathrm{cm}^{2}$ (IQR: $0.83-1.01)$.

The median Z-scores for FN and LS were 0.00 (IQR: -0.7 to +0.8 ) and -0.40 (IQR: -1.2 to +0.3 ), respectively. One hundred and nine patients $(40.2 \%)$ had abnormal Zscores for either FN or LS; abnormal Z-scores for FN and LS were detected in $55(20.3 \%)$ and 89 patients (32.8\%), respectively. One hundred and thirty-eight patients (50.9\%) had discordant Z-scores between FN and LS; only 35 patients $(12.9 \%)$ had abnormal Z-scores for both FN and LS.

The median T-scores for FN and LS were - 0.30 (IQR: -1.0 to +0.5 ) and -0.80 (IQR: -1.5 to +0.2 ), respectively. 
Table 2. Univariate and multivariate analyses on factors associated with higher bone mineral densities (above median) for lumbar spine, by stepwise logistic regression

\begin{tabular}{|c|c|c|c|c|c|c|}
\hline & \multicolumn{3}{|c|}{ Univariate analysis } & \multicolumn{3}{|c|}{ Multivariate analysis } \\
\hline & OR & $95 \% \mathrm{CI}$ for OR & $p$ & OR & $95 \%$ CI for OR & $p$ \\
\hline Age at breast cancer diagnosis & 0.778 & $0.560-1.082$ & 0.1358 & & & \\
\hline$\leq 35$ years & 1 & - & - & & & \\
\hline $36-40$ years & 0.580 & $0.266-1.264$ & 0.1708 & & & \\
\hline $41-45$ years & 0.540 & $0.263-1.110$ & 0.0936 & & & \\
\hline Age at time of study entry & 0.681 & $0.503-0.924$ & 0.0135 & & & \\
\hline$\leq 40$ years & 1 & - & - & & & \\
\hline $41-45$ years & 1.207 & $0.521-2.796$ & 0.6608 & & & \\
\hline $46-50$ years & 0.603 & $0.278-1.306$ & 0.1994 & & & \\
\hline$>50$ years & 0.415 & $0.137-1.254$ & 0.1191 & & & \\
\hline Education & 1.108 & $0.797-1.542$ & 0.5417 & & & \\
\hline Primary & 1 & - & - & & & \\
\hline Secondary & 1.081 & $0.562-2.080$ & 0.8146 & & & \\
\hline Tertiary & 1.231 & $0.473-3.200$ & 0.6701 & & & \\
\hline Higher qualification & 1.429 & $0.476-4.286$ & 0.5246 & & & \\
\hline Marital status & 0.780 & $0.494-1.232$ & 0.2873 & & & \\
\hline Single & 1 & - & - & & & \\
\hline Married/partner & 1.294 & $0.665-2.518$ & 0.4472 & & & \\
\hline Divorced/separated & 0.807 & $0.243-2.675$ & 0.7529 & & & \\
\hline Widowed & - & - & 0.9794 & & & \\
\hline Employment & 0.904 & $0.696-1.174$ & 0.4487 & & & \\
\hline Full-time employment & 1 & - & - & & & \\
\hline Part-time employment & 0.440 & $0.215-0.904$ & 0.0247 & & & \\
\hline Unemployed/retired & 0.834 & $0.494-1.408$ & 0.4977 & & & \\
\hline Family income & 1.649 & $1.181-2.302$ & 0.0033 & 1.573 & $1.091-2.268$ & 0.0152 \\
\hline$<$ HKD 5,000 & 1 & - & - & & & \\
\hline HKD 5,000-25,000 & 1.446 & $0.604-3.461$ & 0.4079 & & & \\
\hline HKD $25,000-50,000$ & 2.862 & $1.141-7.180$ & 0.0251 & & & \\
\hline HKD 50,000 & 3.238 & $0.943-11.117$ & 0.0619 & & & \\
\hline Height at diagnosis $\geq$ median & 2.354 & $1.446-3.833$ & 0.0006 & 2.975 & $1.723-5.137$ & $<0.0001$ \\
\hline Weight at study entry $\geq$ median & 2.510 & $1.539-4.095$ & 0.0002 & & & \\
\hline Height at study entry $\geq$ median & 2.021 & $1.246-3.279$ & 0.0044 & & & \\
\hline BMI at study entry (according to HK BMI) & 1.584 & $1.212-2.069$ & 0.0008 & & & \\
\hline Underweight $(<18.5)$ & 1 & - & - & & & \\
\hline Normal $(18.5-22.9)$ & 1.717 & $0.433-6.801$ & 0.4416 & & & \\
\hline Overweight (23.0-24.9) & 3.627 & $0.873-15.062$ & 0.0762 & & & \\
\hline Obese $(>25)$ & 4.083 & $1.007-16.553$ & 0.0488 & & & \\
\hline Stage of breast cancer & 1.333 & $0.892-1.990$ & 0.1607 & & & \\
\hline Stage I & 1 & - & - & & & \\
\hline Stage II & 1.146 & $0.678-1.939$ & 0.6106 & & & \\
\hline Stage III & 2.142 & $0.853-5.378$ & 0.1047 & & & \\
\hline \multicolumn{7}{|l|}{ Breast surgery } \\
\hline Lumpectomy vs. mastectomy & 0.597 & $0.360-0.989$ & 0.0454 & & & \\
\hline Axillary lymph node dissection vs. nil & 0.316 & $0.032-3.080$ & 0.3215 & & & \\
\hline Received adjuvant radiotherapy & 1.457 & $0.877-2.422$ & 0.1461 & & & \\
\hline Adjuvant chemotherapy regimen & 1.078 & $0.742-1.566$ & 0.6936 & & & \\
\hline Anthracycline-containing & 1 & - & - & & & \\
\hline Anthracycline- and taxane-containing & 1.308 & $0.755-2.267$ & 0.3376 & & & \\
\hline Others (taxane-containing, non-anthracycline + & & & & & & \\
\hline non-taxane containing) & 0.923 & $0.379-2.246$ & 0.8592 & & & \\
\hline Duration of adjuvant chemotherapy $>64$ days & 0.800 & $0.480-1.333$ & 0.3907 & & & \\
\hline \multicolumn{7}{|c|}{ Time since last adjuvant treatment (only include chemotherapy/radiotherapy or trastuzumab) } \\
\hline " 3 to $<5$ years" vs. " 5 to $<10$ years" & 0.549 & $0.337-0.892$ & 0.0155 & 0.435 & $0.250-0.757$ & 0.0032 \\
\hline Ever received adjuvant tamoxifen & 0.636 & $0.363-1.114$ & 0.1137 & & & \\
\hline On adjuvant tamoxifen at study entry & 1.637 & $1.004-2.669$ & 0.0480 & & & \\
\hline Received adjuvant trastuzumab & 0.614 & $0.144-2.621$ & 0.5100 & & & \\
\hline Use of traditional Chinese medicine since diagnosis & 1.617 & $0.955-2.739$ & 0.0737 & & & \\
\hline Having experienced chemotherapy-related amenorrhoea & 1.250 & $0.539-2.899$ & 0.6025 & & & \\
\hline \multicolumn{7}{|l|}{ Menopausal status at study entry } \\
\hline Peri-/postmenopausal vs. premenopausal & 0.431 & $0.262-0.708$ & 0.0009 & 0.443 & $0.255-0.768$ & 0.0038 \\
\hline Menopause before age 45 & 0.488 & $0.291-0.818$ & 0.0065 & & & \\
\hline
\end{tabular}


Table 3. Univariate and multivariate analyses on factors associated with higher bone mineral densities (above median) for femoral neck, by stepwise logistic regression

\begin{tabular}{|c|c|c|c|c|c|c|}
\hline & \multicolumn{3}{|c|}{ Univariate analysis } & \multicolumn{3}{|c|}{ Multivariate analysis } \\
\hline & OR & $95 \%$ CI for OR & $p$ & OR & $95 \%$ CI for OR & $p$ \\
\hline Age at breast cancer diagnosis & 0.800 & $0.576-1.112$ & 0.1848 & & & \\
\hline$\leq 35$ years & 1 & - & - & & & \\
\hline $36-40$ years & 0.448 & $0.204-0.985$ & 0.0457 & & & \\
\hline $41-45$ years & 0.510 & $0.246-1.057$ & 0.0702 & & & \\
\hline Age at time of study entry & 0.767 & $0.569-1.035$ & 0.0830 & & & \\
\hline$\leq 40$ years & 1 & - & - & & & \\
\hline $41-45$ years & 1.294 & $0.561-2.983$ & 0.5451 & & & \\
\hline $46-50$ years & 0.684 & $0.317-1.476$ & 0.3330 & & & \\
\hline$>50$ years & 0.679 & $0.231-1.994$ & 0.4810 & & & \\
\hline Education & 0.990 & $0.712-1.376$ & 0.9519 & & & \\
\hline Primary & 1 & - & - & & & \\
\hline Secondary & 0.905 & $0.474-1.739$ & 0.7648 & & & \\
\hline Tertiary & 0.765 & $0.294-1.995$ & 0.5841 & & & \\
\hline Higher qualification & 1.196 & $0.399-3.585$ & 0.7498 & & & \\
\hline Marital status & 0.869 & $0.553-1.366$ & 0.5426 & & & \\
\hline Single & 1 & - & - & & & \\
\hline Married/partner & 1.270 & $0.653-2.470$ & 0.4816 & & & \\
\hline Divorced/separated & 0.807 & $0.243-2.675$ & 0.7259 & & & \\
\hline Widowed & 0.303 & $0.031-2.941$ & 0.3029 & & & \\
\hline Employment & 0.827 & $0.636-1.075$ & 0.1578 & & & \\
\hline Full-time employment & 1 & - & - & & & \\
\hline Part-time employment & 0.570 & $0.284-1.145$ & 0.1143 & & & \\
\hline Unemployed/retired & 0.691 & $0.409-1.168$ & 0.1678 & & & \\
\hline Family income & 1.216 & $0.882-1.675$ & 0.2321 & & & \\
\hline$<$ HKD 5,000 & 1 & - & - & & & \\
\hline HKD 5,000-25,000 & 1.088 & $0.471-2.515$ & 0.8439 & & & \\
\hline HKD $25,000-50,000$ & 0.985 & $0.407-2.384$ & 0.9736 & & & \\
\hline HKD 50,000 & 3.267 & $0.909-11.743$ & 0.0698 & & & \\
\hline Weight at diagnosis $\geq$ median & 2.835 & $1.732-4.640$ & $<0.0001$ & & & \\
\hline Height at diagnosis $\geq$ median & 1.213 & $0.753-1.955$ & 0.4268 & & & \\
\hline Weight at study entry $\geq$ median & 3.033 & $1.848-4.977$ & $<0.0001$ & & & \\
\hline Height at study entry $\geq$ median & 1.327 & $0.823-2.140$ & 0.2454 & & & \\
\hline BMI at study entry (according to HK BMI) & 1.999 & $1.511-2.643$ & $<0.0001$ & 2.156 & $1.599-2.907$ & $<0.0001$ \\
\hline Underweight $(<18.5)$ & 1 & - & - & & & \\
\hline Normal (18.5-22.9) & 2.423 & $0.500-11.732$ & 0.2715 & & & \\
\hline Overweight (23.0-24.9) & 6.562 & $1.301-33.086$ & 0.0227 & & & \\
\hline Obese $(>25)$ & 8.999 & $1.816-44.586$ & 0.0071 & & & \\
\hline Stage of breast cancer & 0.919 & $0.617-1.368$ & 0.6759 & & & \\
\hline Stage I & 1 & - & - & & & \\
\hline Stage II & 0.804 & $0.476-1.359$ & 0.4154 & & & \\
\hline Stage III & 0.987 & $0.405-2.407$ & 0.9771 & & & \\
\hline \multicolumn{7}{|l|}{ Breast surgery } \\
\hline Lumpectomy vs. mastectomy & 0.777 & $0.471-1.283$ & 0.3244 & & & \\
\hline Axillary lymph node dissection vs. nil & - & - & 0.9812 & & & \\
\hline Received adjuvant radiotherapy & 0.978 & $0.591-1.619$ & 0.9304 & & & \\
\hline Adjuvant chemotherapy regimen & 1.202 & $0.827-1.749$ & 0.3352 & & & \\
\hline Anthracycline-containing & 1 & - & - & & & \\
\hline Anthracycline- and taxane-containing & 1.017 & $0.640-1.915$ & 0.7159 & & & \\
\hline \multicolumn{7}{|l|}{ Others (taxane-containing, non-anthracycline +} \\
\hline non-taxane containing) & 1.599 & $0.650-3.932$ & 0.3064 & & & \\
\hline Duration of adjuvant chemotherapy $>64$ days & 0.747 & $0.448-1.246$ & 0.2634 & & & \\
\hline \multicolumn{7}{|c|}{ Time since last adjuvant treatment (only include chemotherapy/radiotherapy or trastuzumab) } \\
\hline " 3 to $<5$ years" vs. " 5 to $<10$ years" & 0.659 & $0.406-1.069$ & 0.0909 & & & \\
\hline Ever received adjuvant tamoxifen & 0.456 & $0.257-0.808$ & 0.0072 & 0.452 & $0.243-0.841$ & 0.0121 \\
\hline On adjuvant tamoxifen at study entry & 1.131 & $0.696-1.838$ & 0.6182 & & & \\
\hline Received adjuvant trastuzumab & 3.213 & $0.637-16.209$ & 0.1576 & & & \\
\hline Use of traditional Chinese medicine since diagnosis & 1.131 & $0.671-1.907$ & 0.6434 & & & \\
\hline Having experienced chemotherapy-related amenorrhoea & 1.250 & $0.539-2.899$ & 0.6025 & & & \\
\hline \multicolumn{7}{|l|}{ Menopausal status at study entry } \\
\hline Peri-/postmenopausal vs. premenopausal & 0.431 & $0.262-0.708$ & 0.0009 & 0.353 & $0.205-0.609$ & 0.0002 \\
\hline Menopause before age 45 & 0.839 & $0.506-1.390$ & 0.4944 & & & \\
\hline
\end{tabular}




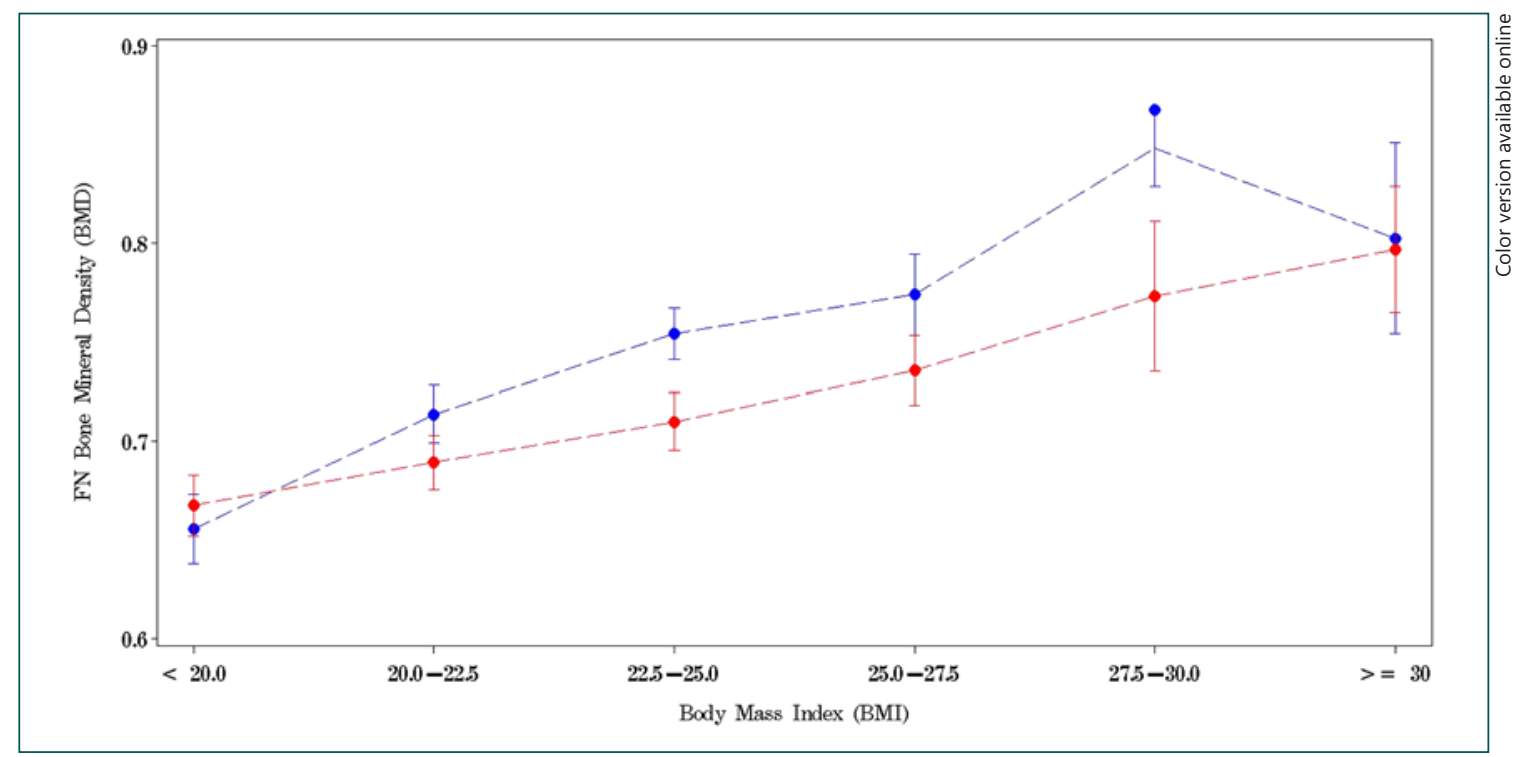

Fig. 1. Bone mineral density of femoral neck (FN) versus body mass index. Blue line, pre-/perimenopausal; red line, postmenopausal.

Osteopenia/osteoporosis of either FN or LS occurred in 136 patients (50.2\%); 67 patients $(24.7 \%)$ had osteopenia/osteoporosis of FN and $122(45.0 \%)$ had osteopenia/ osteoporosis of LS. Ninety-six patients (35.4\%) had discordant $\mathrm{T}$-scores between FN and LS; 53 patients (19.6\%) had osteopenia/osteoporosis for both FN and LS. A total of 17 patients (6.3\%) had osteoporosis at LS, and no patient was detected to have osteoporosis at FN. FRAX scores ranged from 0 to $4 \%$, with a median score of $0.2 \%$.

\section{Analysis for Risk Factors Associated with \\ Abnormalities Detected in DXA}

Univariate and multivariate analyses on factors associated with higher BMD (defined as above median BMD) of LS are listed in Table 2. Patients with higher family income $(\mathrm{OR}=1.573,95 \% \mathrm{CI}=1.091-2.268, p=0.0152)$ and having taller stature $(\mathrm{OR}=2.975,95 \% \mathrm{CI}=1.723-5.137, p<0.0001)$ were significantly associated with higher BMD, while longer time interval since last adjuvant treatment (excluding endocrine therapy) $(\mathrm{OR}=0.435,95 \% \mathrm{CI}=0.250-0.757$, $p=0.0032)$ and peri-/postmenopausal status ( $\mathrm{OR}=0.443$, $95 \% \mathrm{CI}=0.255-0.768, p=0.0038$ ) were significantly and inversely associated with higher BMD.

Table 3 shows the outcomes of univariate and multivariate analyses on factors associated with higher BMD of FN. Higher BMI (OR $=2.156,95 \% \mathrm{CI}=1.599-2.907, p<$ 0.0001 ) was significantly associated with higher BMD (Fig. 1), while having ever received adjuvant tamoxifen $(\mathrm{OR}=0.452,95 \% \mathrm{CI}=0.243-0.841, p=0.0121)$ and peri- $/$ postmenopausal status $(\mathrm{OR}=0.353,95 \% \mathrm{CI}=0.205$ $0.609, p=0.0002$ ) were significantly and inversely associated with higher BMD.
Univariate and multivariate analyses on factors associated with abnormal Z-scores of FN and/or LS are shown in Table 4. Being married/having partner/divorced/separated was shown to be significantly associated with higher risk of abnormal Z-scores (OR $=1.869,95 \% \mathrm{CI}=1.203$ $3.166, p=0.0312$ ), while higher weight at study entry was significantly associated with lower risk of abnormal Zscores $(\mathrm{OR}=0.182,95 \% \mathrm{CI}=0.106-0.312, p<0.0001)$.

Table 5 illustrates the outcomes of univariate and multivariate analyses on factors associated with osteopenia/ osteoporosis of FN and/or LS. Taller body stature $(\mathrm{OR}=$ $0.510,95 \% \mathrm{CI}=0.303-0.858, p=0.0111)$ and higher $\mathrm{BMI}$ $(\mathrm{OR}=0.491,95 \% \mathrm{CI}=0.366-0.658, p<0.0001)$ at study entry were significantly associated with lower risk of osteopenia/osteoporosis. Longer duration of adjuvant chemotherapy $(\mathrm{OR}=1.754,95 \% \mathrm{CI}=1.004-3.063, p=$ 0.0482 ) and peri-/postmenopausal status at study entry $(\mathrm{OR}=2.502,95 \% \mathrm{CI}=1.463-4.280, p=0.0008)$ were significantly associated with lower risk of osteopenia/osteoporosis.

Table 6 shows the factors that were identified on univariate and multivariate analyses to be associated with increased risk of hip fracture based on FRAX score. Higher BMI $(\mathrm{OR}=0.498,95 \% \mathrm{CI}=0.370-0.669, p<0.0001)$ was significantly associated with lower fracture risk while having undergone lumpectomy $(\mathrm{OR}=1.918,95 \% \mathrm{CI}=$ 1.099-3.349, $p=0.0220)$, and longer time interval since last adjuvant treatment $(\mathrm{OR}=1.784,95 \% \mathrm{CI}=1.053-$ 3.022, $p=0.0313)$ and peri-/postmenopausal status at study entry $(\mathrm{OR}=1.985,95 \% \mathrm{CI}=1.162-3.391, p=$ 0.0121 ) were significantly associated with higher risk of fracture. 
Table 4. Univariate and multivariate analyses on factors associated with Z-scores $(\leq-1)$ of femoral neck and/or lumbar spine, by stepwise logistic regression

\begin{tabular}{|c|c|c|c|c|c|c|}
\hline & \multicolumn{3}{|c|}{ Univariate analysis } & \multicolumn{3}{|c|}{ Multivariate analysis } \\
\hline & OR & $95 \%$ CI for OR & $p$ & OR & $95 \%$ CI for OR & $p$ \\
\hline Age at breast cancer diagnosis & 0.890 & $0.639-1.240$ & 0.4914 & & & \\
\hline$\leq 35$ years & 1 & - & - & & & \\
\hline $36-40$ years & 2.154 & $0.972-4.773$ & 0.0588 & & & \\
\hline $41-45$ years & 1.113 & $0.529-2.344$ & 0.7773 & & & \\
\hline Age at time of study entry & 0.853 & $0.631-1.153$ & 0.3009 & & & \\
\hline$\leq 40$ years & 1 & - & - & & & \\
\hline $41-45$ years & 0.730 & $0.316-1.686$ & 0.4617 & & & \\
\hline $46-50$ years & 0.806 & $0.373-1.741$ & 0.5824 & & & \\
\hline$>50$ years & 0.400 & $0.125-1.277$ & 0.1219 & & & \\
\hline Education & 1.484 & $1.055-2.089$ & 0.0235 & & & \\
\hline Primary & 1 & - & - & & & \\
\hline Secondary & 2.237 & $1.066-4.695$ & 0.0332 & & & \\
\hline Tertiary & 2.870 & $1.039-7.927$ & 0.0419 & & & \\
\hline Higher qualification & 3.091 & $0.982-9.734$ & 0.0538 & & & \\
\hline Marital status & 1.739 & $1.074-2.815$ & 0.0245 & 1.869 & $1.203-3.166$ & 0.0312 \\
\hline Single & 1 & - & - & & & \\
\hline Married/partner & 1.317 & $0.655-2.650$ & 0.4394 & & & \\
\hline Divorced/separated & 1.750 & $0.527-5.812$ & 0.3608 & & & \\
\hline Widowed & - & - & 0.9788 & & & \\
\hline Employment & 0.886 & $0.678-1.157$ & 0.3736 & & & \\
\hline Full-time employment & 1 & - & - & & & \\
\hline Part-time employment & 1.154 & $0.277-2.306$ & 0.6854 & & & \\
\hline Unemployed/retired & 0.776 & $0.453-1.329$ & 0.3559 & & & \\
\hline Family income & 1.022 & $0.739-1.413$ & 0.8959 & & & \\
\hline$<$ HKD 5,000 & 1 & - & - & & & \\
\hline HKD 5,000-25,000 & 1.327 & $0.554-3.179$ & 0.5260 & & & \\
\hline HKD $25,000-50,000$ & 1.311 & $0.523-3.285$ & 0.5639 & & & \\
\hline HKD 50,000 & 1.102 & $0.321-3.783$ & 0.8775 & & & \\
\hline Weight at diagnosis $\geq$ median & 0.249 & $0.148-0.418$ & $<0.0001$ & & & \\
\hline Height at diagnosis $\geq$ median & 0.758 & $0.466-1.233$ & 0.2646 & & & \\
\hline Weight at study entry $\geq$ median & 0.193 & $0.114-0.328$ & $<0.0001$ & 0.182 & $0.106-0.312$ & $<0.0001$ \\
\hline Height at study entry $\geq$ median & 0.577 & $0.353-0.942$ & 0.0278 & & & \\
\hline BMI at study entry (according to HK BMI) & 0.422 & $0.311-0.572$ & $<0.0001$ & & & \\
\hline Underweight $(<18.5)$ & 1 & - & - & & & \\
\hline Normal (18.5-22.9) & 0.281 & $0.058-1.356$ & 0.1139 & & & \\
\hline Overweight (23.0-24.9) & 0.083 & $0.016-0.425$ & 0.0028 & & & \\
\hline Obese $(>25)$ & 0.059 & $0.012-0.299$ & 0.0006 & & & \\
\hline Stage of breast cancer & 0.862 & $0.574-1.296$ & 0.4740 & & & \\
\hline Stage I & 1 & - & - & & & \\
\hline Stage II & 0.950 & $0.558-1.618$ & 0.8510 & & & \\
\hline Stage III & 0.654 & $0.255-1.679$ & 0.3769 & & & \\
\hline \multicolumn{7}{|l|}{ Breast surgery } \\
\hline Lumpectomy vs. mastectomy & 1.601 & $0.950-2.700$ & 0.0774 & & & \\
\hline Axillary lymph node dissection vs. nil & - & - & 0.9822 & & & \\
\hline Received adjuvant radiotherapy & 0.646 & $0.388-1.077$ & 0.0941 & & & \\
\hline Adjuvant chemotherapy regimen & 0.870 & $0.591-1.279$ & 0.4782 & & & \\
\hline Anthracycline-containing & 1 & - & - & & & \\
\hline Anthracycline- and taxane-containing & 1.103 & $0.633-1.920$ & 0.7299 & & & \\
\hline Others (taxane-containing, non-anthracycline + & & & & & & \\
\hline non-taxane containing) & 0.547 & $0.204-1.465$ & 0.2301 & & & \\
\hline Duration of adjuvant chemotherapy $>64$ days & 1.430 & $0.842-2.428$ & 0.1862 & & & \\
\hline \multicolumn{7}{|c|}{ Time since last adjuvant treatment (only include chemotherapy/radiotherapy or trastuzumab) } \\
\hline " 3 to $<5$ years" vs. " 5 to $<10$ years" & 0.913 & $0.559-1.490$ & 0.7151 & & & \\
\hline Ever received adjuvant tamoxifen & 1.139 & $0.644-2.013$ & 0.6555 & & & \\
\hline On adjuvant tamoxifen at study entry & 1.049 & $0.640-1.720$ & 0.8485 & & & \\
\hline Received adjuvant trastuzumab & 1.505 & $0.368-6.149$ & 0.5694 & & & \\
\hline Use of traditional Chinese medicine since diagnosis & 0.789 & $0.460-1.352$ & 0.3887 & & & \\
\hline Having experienced chemotherapy-related amenorrhoea & 0.882 & $0.372-2.094$ & 0.7759 & & & \\
\hline \multicolumn{7}{|c|}{ Menopausal status at study entry } \\
\hline Peri-/postmenopausal vs. premenopausal & 1.458 & $0.883-2.407$ & 0.1408 & & & \\
\hline Menopause before age 45 & 1.446 & $0.867-2.410$ & 0.1575 & & & \\
\hline
\end{tabular}


Table 5. Univariate and multivariate analyses on factors associated with osteopenia/osteoporosis of femoral neck and/or lumbar spine, by stepwise logistic regression

\begin{tabular}{|c|c|c|c|c|c|c|}
\hline & \multicolumn{3}{|c|}{ Univariate analysis } & \multicolumn{3}{|c|}{ Multivariate analysis } \\
\hline & OR & $95 \%$ CI for OR & $p$ & OR & $95 \%$ CI for OR & $p$ \\
\hline Age at breast cancer diagnosis & 1.393 & $0.999-1.943$ & 0.0504 & & & \\
\hline$\leq 35$ years & 1 & - & - & & & \\
\hline $36-40$ years & 2.675 & $1.192-6.003$ & 0.0171 & & & \\
\hline $41-45$ years & 2.534 & $1.196-5.372$ & 0.0153 & & & \\
\hline Age at time of study entry & 1.339 & $0.999-1.809$ & 0.0569 & & & \\
\hline$\leq 40$ years & 1 & - & - & & & \\
\hline 41-45 years & 0.927 & $0.401-2.141$ & 0.8590 & & & \\
\hline $46-50$ years & 1.523 & $0.703-3.298$ & 0.2585 & & & \\
\hline$>50$ years & 2.000 & $0.672-5.951$ & 0.2128 & & & \\
\hline Education & 1.014 & $0.730-1.410$ & 0.9330 & & & \\
\hline Primary & 1 & - & - & & & \\
\hline Secondary & 1.235 & $0.642-2.375$ & 0.5275 & & & \\
\hline Tertiary & 0.914 & $0.351-2.385$ & 0.8546 & & & \\
\hline Higher qualification & 1.143 & $0.383-3.412$ & 0.8109 & & & \\
\hline Marital status & 1.420 & $0.894-2.255$ & 0.1374 & & & \\
\hline Single & 1 & - & - & & & \\
\hline Married/partner & 0.935 & $0.482-1.815$ & 0.8429 & & & \\
\hline Divorced/separated & 1.500 & $0.453-4.965$ & 0.5068 & & & \\
\hline Widowed & - & - & 0.9791 & & & \\
\hline Employment & 0.992 & $0.764-1.288$ & 0.9510 & & & \\
\hline Full-time employment & 1 & - & - & & & \\
\hline Part-time employment & 1.694 & $0.840-3.417$ & 0.1409 & & & \\
\hline Unemployed/retired & 0.968 & $0.574-1.633$ & 0.9027 & & & \\
\hline Family income & 0.794 & $0.576-1.096$ & 0.1604 & & & \\
\hline$<$ HKD 5,000 & 1 & - & - & & & \\
\hline HKD 5,000-25,000 & 0.786 & $0.338-1.830$ & 0.5771 & & & \\
\hline HKD $25,000-50,000$ & 0.682 & $0.280-1.660$ & 0.3992 & & & \\
\hline HKD 50,000 & 0.428 & $0.127-1.441$ & 0.1705 & & & \\
\hline Weight at diagnosis $\geq$ median & 0.353 & $0.216-0.578$ & $<0.0001$ & & & \\
\hline Height at diagnosis $\geq$ median & 0.613 & $0.379-0.990$ & 0.0454 & & & \\
\hline Weight at study entry $\geq$ median & 0.310 & $0.189-0.510$ & $<0.0001$ & & & \\
\hline Height at study entry $\geq$ median & 0.527 & $0.325-0.854$ & 0.0092 & 0.510 & $0.303-0.858$ & 0.0111 \\
\hline BMI at study entry (according to HK BMI) & 0.537 & $0.408-0.707$ & $<0.0001$ & 0.491 & $0.366-0.658$ & $<0.0001$ \\
\hline Underweight $(<18.5)$ & 1 & - & - & & & \\
\hline Normal (18.5-22.9) & 0.370 & $0.077-1.791$ & 0.2168 & & & \\
\hline Overweight (23.0-24.9) & 0.152 & $0.030-0.768$ & 0.0227 & & & \\
\hline Obese $(>25)$ & 0.117 & $0.024-0.581$ & 0.0087 & & & \\
\hline Stage of breast cancer & 0.945 & $0.635-1.406$ & 0.7785 & & & \\
\hline Stage I & 1 & - & - & & & \\
\hline Stage II & 1.158 & $0.685-1.956$ & 0.5838 & & & \\
\hline Stage III & 0.698 & $0.283-1.726$ & 0.4369 & & & \\
\hline \multicolumn{7}{|l|}{ Breast surgery } \\
\hline Lumpectomy vs. mastectomy & 1.498 & $0.905-2.478$ & 0.1159 & & & \\
\hline Axillary lymph node dissection vs. nil & - & - & 0.9813 & & & \\
\hline Received adjuvant radiotherapy & 0.802 & $0.484-1.329$ & 0.3917 & & & \\
\hline Adjuvant chemotherapy regimen & 0.923 & $0.635-1.341$ & 0.6737 & & & \\
\hline Anthracycline-containing & 1 & - & - & & & \\
\hline Anthracycline- and taxane-containing & 0.967 & $0.559-1.672$ & 0.9035 & & & \\
\hline Others (taxane-containing, non-anthracycline + & & & & & & \\
\hline non-taxane containing) & 0.806 & $0.331-1.961$ & 0.6345 & & & \\
\hline Duration of adjuvant chemotherapy $>64$ days & 1.687 & $1.007-2.825$ & 0.0471 & 1.754 & $1.004-3.063$ & 0.0482 \\
\hline \multicolumn{7}{|c|}{ Time since last adjuvant treatment (only include chemotherapy/radiotherapy or trastuzumab) } \\
\hline " 3 to $<5$ years" vs. " 5 to $<10$ years" & 1.416 & $0.875-2.294$ & 0.1571 & & & \\
\hline Ever received adjuvant tamoxifen & 1.393 & $0.797-2.434$ & 0.2443 & & & \\
\hline On adjuvant tamoxifen at study entry & 0.683 & $0.420-1.112$ & 0.1255 & & & \\
\hline Received adjuvant trastuzumab & 1.679 & $0.393-7.171$ & 0.4840 & & & \\
\hline Use of traditional Chinese medicine since diagnosis & 0.800 & $0.474-1.350$ & 0.4028 & & & \\
\hline Having experienced chemotherapy-related amenorrhoea & 0.686 & $0.293-1.603$ & 0.3842 & & & \\
\hline \multicolumn{7}{|l|}{ Menopausal status at study entry } \\
\hline Peri-/postmenopausal vs. premenopausal & 2.205 & $1.343-3.622$ & 0.0018 & 2.502 & $1.463-4.280$ & 0.0008 \\
\hline Menopause before age 45 & 1.630 & $0.979-2.713$ & 0.0601 & & & \\
\hline
\end{tabular}


Table 6. Univariate and multivariate analyses on factors associated with increased risk of hip fracture based on FRAX ${ }^{\circledR}$ score

\begin{tabular}{|c|c|c|c|c|c|c|}
\hline & \multicolumn{3}{|c|}{ Univariate analysis } & \multicolumn{3}{|c|}{ Multivariate analysis } \\
\hline & OR & $95 \% \mathrm{CI}$ for OR & $p$ & OR & $95 \% \mathrm{CI}$ for OR & $p$ \\
\hline Age at breast cancer diagnosis & 1.220 & $0.875-1.702$ & 0.2416 & & & \\
\hline$\leq 35$ years & 1 & - & - & & & \\
\hline $36-40$ years & 2.195 & $0.979-4.921$ & 0.0564 & & & \\
\hline $41-45$ years & 1.893 & $0.893-4.014$ & 0.0961 & & & \\
\hline Age at time of study entry & 1.334 & $0.985-1.808$ & 0.0629 & & & \\
\hline$\leq 40$ years & 1 & - & - & & & \\
\hline 41-45 years & 0.696 & $0.299-1.623$ & 0.4019 & & & \\
\hline $46-50$ years & 1.117 & $0.516-2.417$ & 0.7797 & & & \\
\hline$>50$ years & 2.411 & $0.797-7.288$ & 0.1191 & & & \\
\hline Education & 0.930 & $0.667-1.297$ & 0.6711 & & & \\
\hline Primary & 1 & - & - & & & \\
\hline Secondary & 1.012 & $0.525-1.953$ & 0.9704 & & & \\
\hline Tertiary & 1.346 & $0.517-3.505$ & 0.5426 & & & \\
\hline Higher qualification & 0.625 & $0.625-1.960$ & 0.4203 & & & \\
\hline Marital status & 1.088 & $0.692-1.711$ & 0.7135 & & & \\
\hline Single & 1 & - & - & & & \\
\hline Married/partner & 0.952 & $0.489-1.853$ & 0.8846 & & & \\
\hline Divorced/separated & 1.060 & $0.325-3.456$ & 0.9237 & & & \\
\hline Widowed & 1.817 & $0.275-12.025$ & 0.5356 & & & \\
\hline Employment & 1.101 & $0.847-1.432$ & 0.4715 & & & \\
\hline Full-time employment & 1 & - & - & & & \\
\hline Part-time employment & 2.431 & $1.200-4.923$ & 0.0136 & & & \\
\hline Unemployed/retired & 1.188 & $0.699-2.018$ & 0.5252 & & & \\
\hline Family income & 0.989 & $0.718-1.362$ & 0.9457 & & & \\
\hline$<$ HKD 5,000 & 1 & - & - & & & \\
\hline HKD 5,000-25,000 & 1.619 & $0.677-3.873$ & 0.2790 & & & \\
\hline HKD $25,000-50,000$ & 1.844 & $0.738-4.606$ & 0.1902 & & & \\
\hline HKD 50,000 & 0.675 & $0.183-2.481$ & 0.5535 & & & \\
\hline Weight at diagnosis $\geq$ median & 0.409 & $0.250-0.668$ & 0.0004 & & & \\
\hline Height at diagnosis $\geq$ median & 0.806 & $0.499-1.301$ & 0.3770 & & & \\
\hline Weight at study entry $\geq$ median & 0.389 & $0.238-0.637$ & 0.0002 & & & \\
\hline Height at study entry $\geq$ median & 0.655 & $0.404-1.060$ & 0.0848 & & & \\
\hline BMI at study entry (according to HK BMI) & 0.559 & $0.424-0.738$ & $<0.0001$ & 0.498 & $0.370-0.669$ & $<0.0001$ \\
\hline Underweight $(<18.5)$ & 1 & - & - & & & \\
\hline Normal (18.5-22.9) & 0.272 & $0.056-1.311$ & 0.1046 & & & \\
\hline Overweight (23.0-24.9) & 0.123 & $0.024-0.622$ & 0.0113 & & & \\
\hline Obese $(>25)$ & 0.099 & $0.020-0.493$ & 0.0047 & & & \\
\hline Stage of breast cancer & 1.144 & $0.767-1.708$ & 0.5095 & & & \\
\hline Stage I & 1 & - & - & & & \\
\hline Stage II & 1.165 & $0.686-1.979$ & 0.5710 & & & \\
\hline Stage III & 1.282 & $0.524-3.134$ & 0.5859 & & & \\
\hline \multicolumn{7}{|l|}{ Breast surgery } \\
\hline Lumpectomy vs. mastectomy & 1.828 & $1.091-3.062$ & 0.0220 & 1.918 & $1.099-3.349$ & 0.0220 \\
\hline Axillary lymph node dissection vs. nil & - & - & 0.9717 & & & \\
\hline Received adjuvant radiotherapy & 0.747 & $0.450-1.239$ & 0.2589 & & & \\
\hline Adjuvant chemotherapy regimen & 0.837 & $0.573-1.224$ & 0.3595 & & & \\
\hline Anthracycline-containing & 1 & - & - & & & \\
\hline Anthracycline- and taxane-containing & 0.876 & $0.504-1.522$ & 0.6383 & & & \\
\hline $\begin{array}{l}\text { Others (taxane-containing, non-anthracycline }+ \\
\text { non-taxane containing) }\end{array}$ & 0.662 & $0.264-1.657$ & 0.3783 & & & \\
\hline Duration of adjuvant chemotherapy $>64$ days & 1.398 & $0.832-2.349$ & 0.2055 & & & \\
\hline \multicolumn{7}{|c|}{ Time since last adjuvant treatment (only include chemotherapy/radiotherapy or trastuzumab) } \\
\hline " 3 to $<5$ years" vs. " 5 to $<10$ years" & 1.764 & $1.085-2.869$ & 0.0222 & 1.784 & $1.053-3.022$ & 0.0313 \\
\hline Ever received adjuvant tamoxifen & 1.712 & $0.963-3.045$ & 0.0671 & & & \\
\hline On adjuvant tamoxifen at study entry & 0.727 & $0.445-1.188$ & 0.2037 & & & \\
\hline Received adjuvant trastuzumab & 0.403 & $0.080-2.035$ & 0.2716 & & & \\
\hline Use of traditional Chinese medicine since diagnosis & 0.822 & $0.485-1.394$ & 0.4666 & & & \\
\hline Having experienced chemotherapy-related amenorrhoea & 0.593 & $0.245-1.436$ & 0.2470 & & & \\
\hline \multicolumn{7}{|l|}{ Menopausal status at study entry } \\
\hline Peri-/postmenopausal vs. premenopausal & 1.963 & $1.190-3.237$ & 0.0082 & 1.985 & $1.162-3.391$ & 0.0121 \\
\hline Menopause before age 45 & 1.252 & $0.755-2.077$ & 0.3837 & & & \\
\hline
\end{tabular}




\section{Conclusion}

Clinical follow-up of cancer survivors had previously focused on survival and early detection of cancer recurrence. With the development of appropriate adjuvant therapies, survival outcome of breast cancer patients has increased, and as a result, longer survivals are expected. With increased survival, it is anticipated that long-term toxicities associated with cancer treatments may become more evident; these include effects on physical morbidities and psychosocial symptoms. As the incidence of breast cancer among young Asian females has been noted to be rapidly increasing, information on these aspects among the studied population is of particular interest.

Most of the earlier studies assessing BMD in breast cancer patients had been confined to the first year after adjuvant chemotherapy, in which bone loss was reported to be between 2 and $5 \%$ [11, 13, 15, 17, 18, 20, 27]. There is very limited data on long-term follow-up of bone health status among premenopausal patients after adjuvant cytotoxic treatment. In the study reported by Vehmanen et al. [13], follow-up DXA 5 years after adjuvant chemotherapy revealed BMD loss of $5 \%$ in the $\mathrm{FN}$ and $10 \%$ in the LS. Changes in BMD have been reported to vary according to the menstrual status of women after chemotherapy. While it has been reported that bone loss showed no sign of recovery after chemotherapy, partial recovery of BMD has been reported in $70 \%$ of patients who were treated with a finite period of goserelin [20]. In their follow-up study, Vehmanen et al. [28] reported BMD changes at 10 years to be $5 \%$ for those who continued to have regular menses, while it was three-fold greater for those who had irregular menses or remained amenorrhoeic. However, the fact that the study only included 29 patients who received CMF, a regimen rarely used in contemporary adjuvant protocol nowadays, greatly limited the generalizability of the findings.

Although BMD decrease has been consistently reported after chemotherapy, it could not be ascertained whether such a decrease was related to the natural history of loss of BMD in the general population associated with increasing age, as the BMD of the patients concerned have rarely been compared with women of similar age. To our knowledge, the current study is one of the first to include a large number of premenopausal Chinese patients in order to assess the long-term bone health after modern-day adjuvant chemotherapy. With over $90 \%$ of the studied population having received anthracycline- and/or taxane-containing chemotherapy, the present study has shown that $40 \%$ had abnormal Z-scores for FN and/or LS, while 50\% developed osteopenia/osteoporosis at a relatively young age.
Based on multivariate analyses, the present study has identified a number of factors that were associated with poorer bone health in terms of BMDs, Z-scores, Tscores, and FRAX scores. Higher family income, taller stature, and higher BMI at study entry were associated with higher BMD, while longer time interval since last adjuvant treatment, peri-/postmenopausal status at study entry, and having received adjuvant tamoxifen were associated with lower BMD. In addition, abnormal Z-scores were associated with marital status and higher weight at study entry. Further, taller stature and higher BMI at study entry were associated with a lower risk, while longer duration of adjuvant chemotherapy and peri-/postmenopausal status at study entry were associated with higher risk of osteopenia/osteoporosis. Moreover, having undergone breast-conserving surgery, longer interval since chemotherapy and peri-/ postmenopausal status were associated with higher risk, while higher BMI at study entry was associated with lower risk of hip fracture based on FRAX assessment. Apart from chemotherapy, the use of adjuvant tamoxifen, a selective oestrogen receptor modulator, exerts distinctive bone effects between pre- and postmenopausal women; while it improves bone health in the latter [29], it has been associated with increased bone loss in premenopausal women due to its partial antagonistic effect [30].

The study has a number of limitations. For instance, history of administration of bone medication including bisphosphonates, denosumab, calcium, and vitamin D was not collected. There was also lack of follow-up information on occurrence of fractures and serial assessments of BMD. Nonetheless, the identified potential risk factors associated with poorer bone health provide a good rationale for encouraging lifestyle changes to patients after breast cancer diagnosis [31]. In many developing countries, educational status and economical level are major influential factors of reaching healthcare resources and preventative interventions. As such, interventional studies with lifestyle modifications to improve general health status including aspects on bone health are valuable and may enable the provision of useful information to health care providers to avoid longterm negative impacts of anticancer treatments.

\section{Statement of Ethics}

This research project was designed in accordance with the national law and the World Medical Association Declaration of Helsinki (1964) with its ethical principles for medical research involving human subjects and subsequent amendments. Furthermore, the subjects have given their informed consent. 


\section{Disclosure Statement}

The authors declare no conflicts of interest.

\section{Funding Sources}

This study was supported by the Hong Kong Cancer Fund and Madam Diana Hon Fun Kong Donation for Cancer Research.

\section{Author Contributions}

W.Y. contributed to the study conception, design, writing of the manuscript, acquisition of data, and analysis. C.H.W.Y. and F.K.F.M. participated in the statistical analysis and drafting of the manuscript. E.P., Y.L., J.J.S.S., J.K., R.Y.W.N., C.C.H.Y., and L.L. participated in the data collection and drafting of the manuscript. G.S.L. contributed to the study conception and design and writing of the manuscript. All authors read and approved the final manuscript.

\section{References}

1 Chen W, Zheng R, Zhang S, Zeng H, Zuo T, $\mathrm{Xia} \mathrm{C}$, et al. Cancer incidence and mortality in China in 2013: an analysis based on urbanization level. Chin J Cancer Res. 2017 Feb;29(1): $1-10$.

2 Hong Kong Cancer Registry. Hospital Authority (2017): http://www3.ha.org.hk/cancereg/ (accessed 20 April 2017).

3 Yeo W, Lee HM, Chan A, Chan EY, Chan $\mathrm{MC}$, Chan KW, et al. Risk factors and natural history of breast cancer in younger Chinese women. World J Clin Oncol. 2014 Dec;5(5): 1097-106.

4 Rosenberg SM, Partridge AH. Premature menopause in young breast cancer: effects on quality of life and treatment interventions. J Thorac Dis. 2013 Jun;5 Suppl 1:S55-61.

5 Walshe JM, Denduluri N, Swain SM. Amenorrhea in premenopausal women after adjuvant chemotherapy for breast cancer. J Clin Oncol. 2006 Dec;24(36):5769-79.

6 Yeo W, Mo FK, Pang E, Suen JJ, Koh J, Loong $\mathrm{HH}$, et al. Profiles of lipids, blood pressure and weight changes among premenopausal Chinese breast cancer patients after adjuvant chemotherapy. BMC Womens Health. 2017 Jul;17(1):55.

7 Yeo W, Mo FK, Pang E, Suen JJ, Koh J, Yip $\mathrm{CH}$, et al. Quality of life of young Chinese breast cancer patients after adjuvant chemotherapy. Cancer Manag Res. 2018 Feb;10: 383-9.

8 Liem GS, Mo FK, Pang E, Suen JJ, Tang NL, Lee KM, et al. Chemotherapy-Related Amenorrhea and Menopause in Young Chinese Breast Cancer Patients: Analysis on Incidence, Risk Factors and Serum Hormone Profiles.PLoSOne.2015Oct;10(10):e0140842.

9 Bruyère $\mathrm{O}$, Bergmann $\mathrm{P}$, Cavalier E, Gielen E, Goemaere S, Kaufman JM, et al. Skeletal health in breast cancer survivors. Maturitas. 2017 Nov; 105:78-82.

10 Bruning PF, Pit MJ, de Jong-Bakker M, van den Ende A, Hart A, van Enk A. Bone mineral density after adjuvant chemotherapy for premenopausal breast cancer. Br J Cancer. 1990 Feb;61(2):308-10.

11 Shapiro CL, Halabi S, Hars V, Archer L, Weckstein D, Kirshner J, et al. Zoledronic acid preserves bone mineral density in premenopausal women who develop ovarian failure due to adjuvant chemotherapy: final results from CALGB trial 79809. Eur J Cancer. 2011 Mar;47(5):683-9.

12 Saarto T, Blomqvist C, Välimäki M, Mäkelä P, Sarna S, Elomaa I. Chemical castration induced by adjuvant cyclophosphamide, meth- otrexate, and fluorouracil chemotherapy causes rapid bone loss that is reduced by clodronate: a randomized study in premenopausal breast cancer patients. J Clin Oncol. 1997 Apr;15(4):1341-7.

13 Vehmanen L, Saarto T, Elomaa I, Mäkelä P, Välimäki M, Blomqvist C. Long-term impact of chemotherapy-induced ovarian failure on bone mineral density (BMD) in premenopausal breast cancer patients. The effect of adjuvant clodronate treatment. Eur J Cancer. 2001 Dec;37(18):2373-8.

14 Fuleihan GH, Salamoun M, Mourad YA, Chehal A, Salem Z, Mahfoud Z, et al. Pamidronate in the prevention of chemotherapyinduced bone loss in premenopausal women with breast cancer: a randomized controlled trial. J Clin Endocrinol Metab. 2005 Jun; 90(6):3209-14.

15 Shapiro CL, Manola J, Leboff M. Ovarian failure after adjuvant chemotherapy is associated with rapid bone loss in women with earlystage breast cancer. J Clin Oncol. 2001 Jul; 19(14):3306-11.

16 Vehmanen L, Saarto T, Risteli J, Risteli L, Blomqvist C, Elomaa I. Short-term intermittent intravenous clodronate in the prevention of bone loss related to chemotherapy-induced ovarian failure. Breast Cancer Res Treat. 2004 Sep;87(2):181-8.

17 Hershman DL, McMahon DJ, Crew KD, Cremers S, Irani D, Cucchiara G, et al. Zoledronic acid prevents bone loss in premenopausal women undergoing adjuvant chemotherapy for early-stage breast cancer. J Clin Oncol. 2008 Oct;26(29):4739-45

18 Cameron DA, Douglas S, Brown JE, Anderson RA. Bone mineral density loss during adjuvant chemotherapy in pre-menopausal women with early breast cancer: is it dependent on oestrogen deficiency? Breast Cancer Res Treat. 2010 Oct;123(3):805-14.

19 Hines SL, Mincey BA, Sloan JA, Thomas SP, Chottiner E, Loprinzi CL, et al. Phase III randomized, placebo-controlled, double-blind trial of risedronate for the prevention of bone loss in premenopausal women undergoing chemotherapy for primary breast cancer. J Clin Oncol. 2009 Mar;27(7):1047-53.

20 Fogelman I, Blake GM, Blamey R, Palmer M, Sauerbrei W, Schumacher M, et al. Bone mineral density in premenopausal women treated for node-positive early breast cancer with 2 years of goserelin or 6 months of cyclophosphamide, methotrexate and 5-fluorouracil (CMF). Osteoporos Int. 2003 Dec;14(12): 1001-6.
21 Baim S, Binkley N, Bilezikian JP, Kendler DL, Hans DB, Lewiecki EM, et al. Official Positions of the International Society for Clinical Densitometry and executive summary of the 2007 ISCD Position Development Conference. J Clin Densitom. 2008 Jan-Mar;11(1): 75-91.

22 Langdahl BL. Osteoporosis in premenopausal women. Curr Opin Rheumatol. 2017 Jul; 29(4):410-5.

23 World Health Organization. Technical Report Series: Research on the Menopause. Geneva, Switzerland: WHO Technical Report Series No. 670, 1981.

24 World Health Organization, International Association for the Study of Obesity, International Obesity Task Force. The Asia-Pacific Perspective: Redefining Obesity and Its Treatment. Melbourne, Australia: Health Communications Australia Pty Ltd; 2000.

25 Kanis JA, McCloskey EV, Johansson H, Oden A, Melton LJ 3rd, Khaltaev N. A reference standard for the description of osteoporosis. Bone. 2008 Mar;42(3):467-75.

26 Fracture Risk Assessment Tool $\left(\right.$ FRAX $\left.^{\circledR}\right)$ : https://www.sheffield.ac.uk/FRAX/tool. aspx? country=9 (assessed in March 2019).

27 Hershman DL, McMahon DJ, Crew KD, Shao T, Cremers S, Brafman L, et al. Prevention of bone loss by zoledronic acid in premenopausal women undergoing adjuvant chemotherapy persist up to one year following discontinuing treatment. J Clin Endocrinol Metab. 2010 Feb;95(2):559-66.

28 Vehmanen LK, Elomaa I, Blomqvist CP, Saarto T. The effect of ovarian dysfunction on bone mineral density in breast cancer patients 10 years after adjuvant chemotherapy. Acta Oncol. 2014 Jan;53(1):75-9.

29 Hadji P, Gnant M, Body JJ, Bundred NJ, Brufsky A, Coleman RE, et al. Cancer treatmentinduced bone loss in premenopausal women: a need for therapeutic intervention? Cancer Treat Rev. 2012 Oct;38(6):798-806.

30 Powles TJ, Hickish T, Kanis JA, Tidy A, Ashley S. Effect of tamoxifen on bone mineral density measured by dual-energy $\mathrm{x}$-ray absorptiometry in healthy premenopausal and postmenopausal women. J Clin Oncol. 1996 Jan;14(1):78-84.

31 Rock CL, Flatt SW, Byers TE, Colditz GA, Demark-Wahnefried W, Ganz PA, et al. Results of the exercise and nutrition to enhance recovery and good health for you (ENERGY) trial: a behavioral weight loss intervention in overweight or obese breast cancer survivors. J Clin Oncol. 2015 Oct;33(28):3169-76. 